\title{
Análise comparativa dos resultados da cirurgia do labirinto (Cox-maze) para fibrilação atrial crônica em pacientes com doença valvar mitral reumática ou degenerativa
}

\author{
Renato A. K. KALIL*, Bartira CUNHA*, Álvaro S. ALBRECHT*, Paulo MORENO*, Rogério ABRAHÃO*, \\ Paulo R. PRATES*, João R. M. SANT'ANNA*, Ivo A. NESRALLA*
}

RBCCV 44205-460

Kalil R A K, Cunha B, Albrecht A S, Moreno P, Abrahão R, Prates PR, Sant'Anna J R M, Nesralla I A - Análise comparativa dos resultados da cirurgia do labirinto (Cox-maze) para fibrilação atrial crônica em pacientes com doença valvar mitral reumática ou degenerativa. Rev Bras Cir Cardiovasc 1999;14 (3): 191-99.

RESUMO: Objetivo: Comparar resultados da correção de Fibrilação Atrial (FA) Crônica em Doença Mitral Reumática $(R)$ ou Degenerativa (D) a curto e médio prazos.

Casuística e Métodos: De 1994 a 1997, 57 pacientes foram submetidos à Cirurgia do Labirinto e correção mitral. Oito (20\%) reumáticos eram do sexo masculino; $32(80 \%)$ feminino. Dos degenerativos $8(47 \%)$ eram do sexo masculino; 9 (53\%) femininos, (NS). Idade $47 \pm 11$ anos $R ; 54 \pm 17 \mathrm{D}(p<0,05)$. Diâmetro do AE: 6,1 $\pm 1,1 \mathrm{~cm}$ R e $5,9 \pm 1,2 \mathrm{D}$, (NS).

Resultados: Óbitos: 1 (2,5\%) imediato em R; $2(12 \%)$ hospitalares e $1(7 \%)$ tardio em D (NS). Implante de marcapasso (MP) em $4(10 \%) R$ e $2(17 \%) D(N S)$. Tempos de isquemia: $63 \pm 16$ min $R ; 63 \pm 15$ min $D$, (NS). Circulação extracorpórea: $92 \pm 19$ min R e $96 \pm 23$ min D, (NS). Na alta hospitalar: 3 (10\%) R e nenhum D recebiam medicação antiarrítmica, (NS); 9 (23\%) R e $3(20 \%)$ D recebiam anticoagulantes (NS). Conversão a ritmo sinusal (RS) em 31 (80\%) do grupo R e em 12 (80\%) do D, (NS). Ritmo de MP em 4 (10\%) R, NS. FA incidiu em $4(10 \%) \mathrm{R}$ e em $2(13 \%) \mathrm{D}$, (NS). Ritmo juncional em 1 (7\%) D, (NS). Na última avaliação: RS em 27 (71\%) Re 9 (75\%) D (NS). Ritmo de MP em 4 (10\%) Re 2 (17\%) D, (NS). FA em 6 (16\%) R e nenhum D, (NS). Taquicardia atrial paroxística em 1 (3\%) R e $1(8 \%)$ do grupo D, (NS). À ergometria, realizada em média de $16,2 \pm 11,1$ meses PO por $24(60 \%)$ pacientes $R$ e a $16,4 \pm 10,0$ meses PO para 10 $(59 \%)$ degenerativos, 6 (25\%) R e 1 (10\%) D apresentaram RC adequada (NS). Em $3(12,5 \%)$ R e $6(60 \%)$ $\mathrm{D}$ foi considerada intermediária $(p=0,009)$. RC inadequada foi detectada em $15(62,5 \%)$ pacientes $\mathrm{R}$ e 3 $(30 \%) D^{-}(p=0,09)$.

Conclusão: A etiologia R ou D não afeta a morbimortalidade e os benefícios aos pacientes com valvopatia mitral submetidos à Cirurgia do Labirinto e correção valvar. A recuperação do RS e a presença de arritmias no $\mathrm{PO}$ foi semelhante nos grupos. A resposta cronotrópica ao exercício tende a ser menor no grupo degenerativo.

DESCRITORES: Valva mitral, cirurgia. Fibrilação atrial, cirurgia. Arritmia sinusal, cirurgia. Cardiopatia reumática.

Trabalho realizado no Serviço de Cirurgia Cardiovascular do Instituto de Cardiologia do Rio Grande do Sul. Fundação Universitária de Cardiologia. Porto Alegre, RS, Brasil.

Apresentado ao 26을 Congresso Nacional de Cirurgia Cardíaca. Fortaleza, CE, 8 a 10 de abril, 1999

* Do Serviço de Cirurgia Cardiovascular do Instituto de Cardiologia do Rio Grande do Sul.

Endereço para correspondência: Renato A. K. Kalii. Av. Princesa Isabel, 395-Santana. Porto Alegre, RS, Brasil. CEP 90620-001. Tel: (051) 217-2035, ramal 257/277. Fax: (051) 217-2035. e-mail: pesquisa@cardnet.tche.br 
Kalil R A K, Cunha B, Albrecht A S, Moreno P, Abrahão R, Prates PR, Sant'Anna J R M, Nesralla I A - Análise comparativa dos resultados da cirurgia do labirinto (Cox-maze) para fibrilação atrial crônica em pacientes com doença valvar mitral reumática ou degenerativa. Rev Bras Cir Cardiovasc 1999; 14 (3): 191-99.

\section{INTRODUÇÃO}

A fibrilação atrial (FA) é a mais prevalente das alterações do ritmo cardíaco, atingindo $0,15 \%$ da população em geral (1). Variáveis como a idade e a existência de doença cardíaca estrutural incrementam a incidência da arritmia em cerca de $10 \%$ da população acima de 60 anos e em $40 \%$ a $60 \%$ dos pacientes com valvopatia mitral (2). Neste último caso, representa o principal fator de risco para o desenvolvimento de eventos tromboembólicos (3).

Com o objetivo de corrigir integralmente a arritmia, afastando suas seqüelas detrimentais, COX et al. (4) desenvolveram, em 1987, a Técnica do Labirinto. $O$ procedimento - indicado para o tratamento da fibrilação atrial crônica refratária - teve sua eficácia comprovada ao beneficiar inicialmente uma série de pacientes com FA primária ${ }^{(4)}$. Posteriormente, ao ser evidenciado que a correção da valvopatia mitral por si não altera a prevalência da arritmia (5), o Método do Labirinto passou a ser associado às intervenções cirúrgicas orovalvares.

Os resultados iniciais determinados pela correção simultânea da lesão valvar e da arritmia pela combinação dos dois procedimentos são conhecidos; entretanto, os fatores de risco acrescidos pela associação simultânea das técnicas cirúrgicas, passíveis de alterar a morbimortalidade (pós-operatória) a médio e a longo prazos, ainda não foram definitivamente elucidados ${ }^{(6)}$. Nesse contexto, variáveis pré-operatórias como a etiologia da lesão orovalvar - reumática ou degenerativa - têm sido apontadas, em alguns estudos, como responsáveis por um menor índice de recuperação da contratilidade e do ritmo atrial no pós-operatório ${ }^{(7,8)}$, enquanto que outros autores não atribuíram à patogênese valvar qualquer valor preditivo de morbimortalidade pós-cirúrgica $(9,10)$.

Neste trabalho, os resultados da Cirurgia do Labirinto associada à correção da valvopatia mitral em pacientes com doença cardíaca de etiologia reumática ou degenerativa foram submetidos à análise comparativa, visando-se estabelecer a participação da patogênese valvar na morbimortalidade, bem como, na recuperação do ritmo sinusal no pósoperatório a médio e a longo prazos.

\section{CASUÍSTICA E MÉTODOS}

No período compreendido entre maio de 1994 e maio de 1997, 57 pacientes consecutivos, com FA crônica secundária, submeteram-se ao Procedimento do Labirinto (Cox-maze) simultaneamente à correção da doença valvar mitral. Retrospectivamente, os dados obtidos ao longo de acompanhamento clínico pré e pós-operatórios foram separados em dois grupos - Reumáticos (R) e Degenerativos (D) - para fins de análise comparativa. Eram 40 pacientes reumáticos e $17 \mathrm{com}$ lesão mitral de etiologia degenerativa. Outras doenças orgânicas cardíacas associadas à lesão mitral foram: insuficiência tricúspide, $1(6 \%)$ paciente $D$; dupla lesão aórtica, $1(2,5 \%) R$; cardiopatia isquêmica, $1(6 \%) \mathrm{D}$.

Os critérios de inclusão no estudo, ou seja, de seleção para operação foram, respectivamente, para correção da arritmia e da valvopatia presença de fibrilação atrial crônica refratária por período superior a seis meses em pacientes submetidos pela primeira vez à cirurgia cardíaca e/ou que já tenham apresentado episódio de acidente vascular cerebral; fração de ejeção maior do que $20 \%$ e possibilidade de intervenção mitral de forma eletiva.

\section{Pacientes}

Entre os 40 (70\%) pacientes reumáticos, 8 (20\%) pertenciam ao sexo masculino, 32 (80\%) ao feminino; enquanto que o grupo com doença degenerativa esteve representado por 8 (47\%) homens e $9(53 \%)$ mulheres. A média de idade foi $47 \pm 11$ anos (variação de 20 a 70 anos) no grupo $R$ e de $54 \pm 17$ anos (mínima de 26 e máxima de 70 anos) no grupo D. Na avaliação clínica pré-operatória, 2 (5\%) pacientes com lesão valvar reumática preencheram os critérios de NYHA para a classe funcional I; 5 (12,5\%) para classe II; $27(67,5 \%)$ enquadraram-se na classe III e $6(15 \%)$ na IV. Para o grupo D, observouse a seguinte distribuição: $1(6,0 \%)$ paciente em classe I; 3 (18\%) em classe II; $12(70 \%)$ e 1 (6,0\%) em classes III e IV, nessa ordem. $\mathrm{Na}$ análise ecocardiográfica realizada no período imediatamente anterior à operação, os índices relativos às medidas da fração de ejeção (FE) de 31 (77,5\%) reumáticos e $14(82 \%)$ degenerativos para os quais foi possível obter esses dados, foram, respectivamente, $64,7 \pm 9,2 \%$ e $63,5 \pm 10,3 \%$. As médias de diâmetro de átrio esquerdo ( $\mathrm{AE}$ ) para $33(82,5 \%)$ reumáticos equivaleram a $6,1 \pm 1,1 \mathrm{~cm} ; 5,9 \pm 1,2$ $\mathrm{cm}$, foi o valor medido para $15(88 \%)$ pacientes com lesão valvar degenerativa.

Em relação ao conjunto das características préoperatórias, apenas a variável relativa à idade mostrou diferença significativa entre os grupos etiológicos da lesão valvar $(p<0,05)$, como podese observar na Tabela 1. Nos demais aspectos, os grupo exibiram homogeneidade.

\section{Métodos}

As informações relativas à situação clínica pré e pós-operatória da série em estudo foram extraídas dos protocolos de pesquisa previamente preen- 
Kalil R A K, Cunha B, Albrecht A S, Moreno P, Abrahão R, Prates PR, Sant'Anna J R M, Nesralla I A - Análise comparativa dos resultados da cirurgia do labirinto (Cox-maze) para fibrilação atrial crônica em pacientes com doença valvar mitral reumática ou degenerativa. Rev Bras Cir Cardiovasc 1999; 14 (3): 191-99.

TABELA 1

CARACTERÍSTICAS CLÍNICAS PRÉ-OPERATÓRIAS DOS PACIENTES COM DOENÇA VALVAR REUMÁTICA E DEGENERATIVA

\begin{tabular}{|c|c|c|c|}
\hline CARACTERÍSTICAS & $\boldsymbol{R}$ & $D$ & SIGNIFICÂNCIA \\
\hline$\underset{N}{\text { Demográficas }}$ & $40(70 \%)$ & $17(30 \%)$ & \\
\hline $\begin{array}{c}\text { Sexo masculino } \\
\text { feminino }\end{array}$ & $\begin{array}{l}8(20 \%) \\
32(80 \%)\end{array}$ & $\begin{array}{l}8(47 \%) \\
9(53 \%)\end{array}$ & $\begin{array}{l}\text { NS } \\
\text { NS }\end{array}$ \\
\hline Idade (anos) ${ }^{a}$ & $47 \pm 11$ & $54 \pm 17$ & $0,002^{b}$ \\
\hline $\begin{array}{c}\text { Classe Funcional } \\
\text { I } \\
\text { II } \\
\text { III } \\
\text { IV }\end{array}$ & $\begin{array}{c}2(5 \%) \\
5(12,5 \%) \\
27(67,5 \%) \\
6(15 \%)\end{array}$ & $\begin{array}{c}1(6 \%) \\
3(18 \%) \\
12(70 \%) \\
1(6 \%)\end{array}$ & $\begin{array}{l}\text { NS } \\
\text { NS } \\
\text { NS } \\
\text { NS }\end{array}$ \\
\hline $\begin{array}{l}\text { Índices Ecocardiográficos } \\
\mathrm{N}^{*} \\
\mathrm{FE}^{\mathrm{a}} \\
\mathrm{N}^{*} \\
\text { Diâmetro de } \mathrm{AE}(\mathrm{cm})^{\mathrm{a}}\end{array}$ & $\begin{array}{c}31(77,5 \%) \\
64,7 \pm 9,2 \\
33(82,5 \%) \\
6,1 \pm 1,1\end{array}$ & $\begin{array}{c}14(82 \%) \\
63,5 \pm 10,3 \\
15(88 \%) \\
5,9 \pm 1,2\end{array}$ & $\begin{array}{l}\text { NS } \\
\text { NS }\end{array}$ \\
\hline
\end{tabular}

$\mathrm{R}=$ pacientes com doença valvar reumática $\mathrm{D}=$ pacientes com doença valvar degenerativa; $\mathrm{N}=$ número de pacientes $;{ }^{\mathrm{a}}=\mathrm{v}$ alores expressos com média \pm desvio padrão; ${ }^{\mathrm{b}}=$ estatisticamente significativo; $\mathrm{N}^{*}=$ número de pacientes para o qual foi possível obter informações; $F E$ = fração de ejeção; $A E$ = átrio esquerdo.

chidos ao longo de acompanhamento realizado com os pacientes em ambulatório específico, incluindo avaliações clínicas periódicas, exames de eletrocardiografia, de ergometria e de ecocardiografia. As variáveis intra-operatórias - tempo de isquemia, tempo de circulação extracorpórea, procedimentos cirúrgicos e a informação sobre a etiologia da lesão valvar - foram fornecidas pelos relatórios cirúrgicos. A decisão sobre como classificar a valvopatia em reumática ou degenerativa foi embasada em diagnóstico clínico e no aspecto da alteração valvar.

Ao eletrocardiograma, o ritmo cardíaco foi caracterizado de acordo com o eixo da onda $P$ nos planos frontal e horizontal e com sua morfologia nas 12 derivações. Conhecendo-se as alterações na morfologia da onda $\mathrm{P}$ pela Cirurgia do Labirinto, considerou-se sinusal a onda $P$ com eixo entre ${ }^{-30}$ a +90 no plano frontal, de morfologia plus-minus em $\mathrm{V}_{1}$ e de início positivo em $\mathrm{V}_{5}$ e $\mathrm{V}_{6}$.

É importante salientar as alterações na morfologia da onda $P$, seu achatamento e as modificações na duração que, por vezes, dificultam a análise e a definição do ritmo como atrial ou sinusal, comum no pós-operatório da Cirurgia do Labirinto.

\section{Método do Labirinto}

O procedimento cirúrgico utilizado foi fundamentalmente o mesmo inicialmente descrito por COX et al. (4) e posteriormente modificado, constituindo o método atualmente empregado chamado Cox 3 (11), entretanto sem o emprego de crioablação do seio coronário e das intersecções com os anéis das valvas mitral e tricúspide. Em lugar de crioablação, foi realizada dissecção mais extensa no local, seguida de eletrocoagulação.

\section{Análise Estatística}

Os valores numéricos foram expressos como média \pm desvio padrão. Foi empregado o teste " $t$ " de Student-Fisher para variáveis contínuas e o teste do qui-quadrado para variáveis categóricas, consideram-se $\alpha$-crítico em $5 \%(p<0,05)$.

\section{RESULTADOS}

As variáveis intra-operatórias mensuradas e analisadas neste estudo foram: tempos de circulação extracorpórea e de isquemia miocárdica, assim como os procedimentos cirúrgicos associados à $\mathrm{Ci}$ rurgia do Labirinto.

O tempo de circulação extracorpórea (CEC) foi de $92 \pm 19$ min para os pacientes do grupo $R$ e de $96 \pm 23 \mathrm{~min}$ para os do grupo D (NS); enquanto os intervalos médios de isquemia miocárdica corresponderam a $63 \pm 16 \mathrm{~min}$ para os pacientes $R$ e $D$, respectivamente, (NS).

A valvoplastia mitral de forma isolada foi o procedimento cirúrgico realizado em concomitância com a Técnica do Labirinto em 37 (93\%) pacientes com valvopatia de etiologia reumática e em 14 (82\%) daqueles com lesão do tipo degenerativa. Esteve associada à reconstrução valvar aórtica em 1 (2,5\%) paciente do grupo $\mathrm{R}$; à valvoplastia tricúspide em 1 (6\%), e à revascularização miocárdica, em igual 
Kalil R A K, Cunha B, Albrecht A S, Moreno P, Abrahão R, Prates PR, Sant'Anna J R M, Nesralla I A - Análise comparativa dos resultados da cirurgia do labirinto (Cox-maze) para fibrilação atrial crônica em pacientes com doença valvar mitral reumática ou degenerativa. Rev Bras Cir Cardiovasc 1999; 14 (3): 191-99.

número, $1(6 \%)$, pacientes D. A conservação valvar não foi possível em $2(5 \%) \mathrm{R}$, e em 1 (6\%) D, havendo, nesses casos, colocação de prótese mitral.

No período pós-operatório imediato, abrangendo um tempo de internação de $11 \pm 5$ dias para pacientes $R$ e de $12 \pm 4$ dias para pacientes $D$ (NS), haviam sido registrados $2(12 \%)$ óbitos no grupo $D$ e $1(2,5 \%)$ no grupo R, (NS). A implantação de marcapasso permanente, no mesmo período, foi necessária em 2 (5\%) R, (NS). Na alta hospitalar, $3(10 \%)$ pacientes $\mathrm{R}$ recebiam medicação antiarrítmica (NS), omitindo-se, nesse caso, a informação a cerca do uso de digital por ter sido esta medicação indicada, primariamente, para o tratamento da doença orovalvar. Em $9(23 \%)$ reumáticos e em 3 (20\%) degenerativos houve administração de anticoagulantes (NS). Os porcentuais referentes ao uso de medicação no pós-operatório imediato foram calculados sobre o número de sobreviventes hospitalares. Na Tabela 2 estão discriminados os resultados dos dois analisados à alta hospitalar (Tabela 2).

Em relação ao intervalo compreendido pelo pósoperatório tardio, as variáveis supracitadas - excetuando-se o tempo de internação, excluídos os óbitos hospitalares e os pacientes sem acompanhamento pós-operatório - apresentaram a seguinte distribuição na Tabela 3: o único óbito $(7,0 \%)$, relativo a um período médio de follow up de 17,4 \pm 14,1 meses (R) e de 18,5 $\pm 12,1$ meses (D), (NS), incidiu na população de pacientes degenerativos, (NS); implantação de marcapasso permanente ocorreu em $2(5,0 \%) \mathrm{R}$ e em $2(17,0 \%) \mathrm{D}$, (NS); 7 (18\%) pacientes do grupo R e 1 (8,0\%) do grupo D encontravamse sob controle antiarrítmico (excluindo-se uso de digital), (NS); ao passo que, 8 (21\%) reumáticos e $2(17 \%)$ degenerativos recebiam anticoagulantes, (NS). Na última avaliação pós-operatória, 28 (82\%) pacientes do grupo $R$ e $8(73 \%)$ do $D$, (NS), preencheram os critérios da NYHA compatíveis com a classe I; 6 (18\%) e 1 (9\%), R e D, respectivamente, encontravam-se em classe II; 2 (18\%) pacientes com doença valvar degenerativa achavam-se em classe III, (NS). No Gráfico 1 e na Tabela 3, podese observar o perfil da distribuição dos pacientes $R$ e $D$ nas classes funcionais pré e pós-operatórias, bem como o contraste entre os dois grupos dentro das classificações individuais da NYHA (Tabela 3).

Foi possível mensurar as médias dos diâmetros de átrio esquerdo em 35 (87,5\%) reumáticos e em $11(64,5 \%)$ degenerativos, e os valores medidos corresponderam a $5,4 \pm 1,0 \mathrm{~cm}$ e a $5,0 \pm 0,9 \mathrm{~cm}$ nos grupos R e D, respectivamente, (NS). Os porcentuais relativos à fração de ejeção obtidos para 34 (89\%) pacientes do grupo $R$ e para $11(64,5 \%)$ do $D$ foram de $64,0 \pm 4,7(R)$ e de 63,2 $\pm 6,7$ (D), (NS).

Por ocasião do teste ergométrico realizado à média de 16,8 meses pós-operatórios por 24 (60\%) reumáticos e a 16,4 meses por 10 (59\%) degenerativos - a resposta cronotrópica do exercício (RC) foi considerada adequada (maior do que $85 \%$ da freqüência cardíaca máxima calculada) em 6 (25\%) $\mathrm{R}$ e em 1 (10\%) D, (NS); intermediária (entre 75\% e $85 \%)$ em $3(12,5 \%)$ pacientes reumáticos e em 6 (60\%) degenerativos e inadequada (menor que $75 \%$ ) em $15 R(62,5 \%)$ e $3 D(30 \%)(p=0,09)$, sendo essa a única variável pós-operatória a exibir diferença estatística entre os grupos comparadas $(p=0,009)$. Os índices ecocardiográficos e ergométricos encontram-se discriminados na Tabela 3.

$\mathrm{Na}$ análise eletrocardiográfica, realizada à alta hospitalar, englobando $39(97,5 \%)$ pacientes reumáticos e 15 (88\%) degenerativos, ritmo sinusal foi detectado em 31 (80\%) R e em 12 (80\%) D, (NS); ritmo de marcapasso incidiu apenas no contingente de pacientes reumáticos, 4 (10\%) NS. Em 4 (10\%) pacientes do grupo $R$ e em 2 (13\%) do grupo D, o traçado do ECG mostrou fibrilação atrial (NS); 1 (7\%) paciente D apresentou ritmo juncional, (NS).

Para 38 (95\%) reumáticos e 12 (70\%) degenerativos, submetidos à última avaliação pós-operatória, ritmo sinusal esteve presente em 27 (71\%) e em $9(75 \%)$ pacientes R e D, em ordem de citação, (NS). Quatro (10\%) R e 2 (17\%) D encontravam-se sob comando de marcapasso, (NS); 6 (16\%) pacientes com doença valvar de etiologia reumática

TABELA 2

VARIÁVEIS PÓS-OPERATÓRIAS IMEDIATAS E SUA DISTRIBUIÇÃO SEGUNDO A ETIOLOGIA DA LESÃO VALVAR

\begin{tabular}{lccc}
\hline VARIÁVEIS & $\boldsymbol{R}$ & $\boldsymbol{D}$ & SIGNIFICÂNCIA \\
\hline Óbito & $1(2,5 \%)$ & $2(12 \%)$ & NS \\
Implante de marcapasso $_{\text {Antiarrítmico na alta hospitalar }{ }^{\text {a }}{ }^{*}}$ & $2(5,0 \%)$ & - & NS \\
Anticoagulante na alta hospitalar $^{*}$ & $3(10 \%)$ & - & NS \\
Tempo de internação (dias) $^{b}$ & $9(23 \%)$ & $3(20 \%)$ & NS \\
\hline
\end{tabular}

$\mathrm{R}=$ pacientes com doença valvar reumática; $\mathrm{D}=$ pacientes com doença valvar degenerativa; ${ }^{*}=$ porcentual sobre sobreviventes hospitalares; $^{\mathrm{a}}=$ não inclui digital; ${ }^{\mathrm{b}}=$ valores expressos como média \pm desvio padrão. 
Kalil R A K, Cunha B, Albrecht A S, Moreno P, Abrahão R, Prates PR, Sant'Anna J R M, Nesralla I A - Análise comparativa dos resultados da cirurgia do labirinto (Cox-maze) para fibrilação atrial crônica em pacientes com doença valvar mitral reumática ou degenerativa. Rev Bras Cir Cardiovasc 1999; 14 (3): 191-99.

TABELA 3

VARIÁVEIS PÓS-OPERATÓRIAS TARDIAS E SUA DISTRIBUIÇÃO SEGUNDO A ETIOLOGIA DA LESÃO VALVAR

\begin{tabular}{|c|c|c|c|}
\hline VARIÁVEIS & $\boldsymbol{R}$ & $D$ & SIGNIFICÂNCIA \\
\hline Óbito * & - & $1(7 \%)$ & NS \\
\hline Implante de marcapasso * & $2(5 \%)$ & $2(17 \%)$ & NS \\
\hline Antiarrítmico na última avaliação * a & $7(18 \%)$ & $1(8 \%)$ & NS \\
\hline Anticoagulante na última avaliação * & $8(21 \%)$ & $2(17 \%)$ & NS \\
\hline \multicolumn{4}{|l|}{ Classe Funcional } \\
\hline 1 & $28(82 \%)$ & $8(73 \%)$ & NS \\
\hline II & $6(18 \%)$ & $1(9 \%)$ & NS \\
\hline III & - & $2(18 \%)$ & NS \\
\hline IV & - & - & - \\
\hline Tempo Follow up (meses) * b & $17,4 \pm 14,1$ & $18,5 \pm 12,1$ & NS \\
\hline \multicolumn{4}{|l|}{ Índices ergométricos } \\
\hline $\mathrm{N}^{*}$ & $24(10 \%)$ & $10(59 \%)$ & \\
\hline $\mathrm{RC}$ adequada & $6(25 \%)$ & $1(10 \%)$ & NS \\
\hline $\mathrm{RC}$ intermediária & $3(12,5 \%)$ & $6(60 \%)$ & $0,009^{c}$ \\
\hline$R C$ inadequada & $15(62,5 \%)$ & $3(30 \%)$ & 0,09 \\
\hline \multicolumn{4}{|l|}{ Índices Ecocardiográficos } \\
\hline $\mathrm{N}^{*}$ & $35(87,5 \%)$ & $11(64,5 \%)$ & \\
\hline Diâmetro de $A E(\mathrm{~cm})^{b}$ & $5,4 \pm 1,0$ & $5,0 \pm 0,9$ & NS \\
\hline $\mathrm{N}^{*}$ & $34(89 \%)$ & $11(64,5 \%)$ & \\
\hline FE b & $64,0 \pm 4,7$ & $63,2 \pm 6,7$ & NS \\
\hline
\end{tabular}

$\mathrm{R}=$ pacientes com doença valvar reumática; $\mathrm{D}$ = pacientes com doença valvar degenerativa; * excluídos os óbitos hospitalares e os pacientes sem acompanhamento pós-operatório; ${ }^{\mathrm{a}}=$ não inclui digital; ${ }^{\mathrm{b}}=$ valores expressos como média \pm desvio padrão; $\mathrm{N}^{*}=$

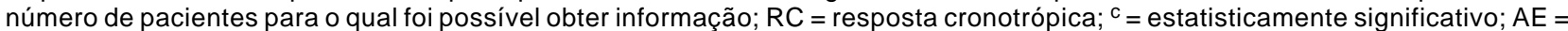
átrio esquerdo; $F E$ = fração de ejeção.

estavam em fibrilação (NS). ECG com Taquicardia Atrial Paroxística foi observado em igual número de pacientes $\mathrm{R}, 1$ (3\%), e D, 1 (8\%), (NS).

A representação gráfica do ritmo cardíaco dos pacientes analisados neste estudo, à alta hospitalar e à última avaliação pós-operatória, estão esboçados nas Figuras 1 e 2, respectivamente.

\section{COMENTÁRIOS}

A técnica cirúrgica do Labirinto - indicada para o tratamento da FAC - foi, inicialmente, utilizada para o manejo da forma primária da arritmia. Os resultados promissores obtidos por COX et al. ${ }^{(4)} \mathrm{em}$ relação à recuperação do ritmo e da contratilidade atriais contribuíram para atestar a eficácia do procedimento e promover sua difusão.

Posteriormente, à medida que a experiência foi sendo acumulada ${ }^{(6)}$, o método operatório passou a ser associado à correção de lesões cardíacas estruturais responsáveis secundariamente pela gênese da arritmia. Entretanto, em algumas séries de pacientes submetidos à combinação cirúrgica, os índices de sucesso iniciais foram algo inferiores aos relatados por Cox, em especial, nas taxas de reversão a ritmo sinusal e de restauração da função atrial $(7,8)$. Nesse contexto, a etiologia da lesão valvar - reumá- tica ou degenerativa - passou a ser investigada como uma variável passível de comprometer os desfechos pós-operatórios.

Nesse estudo, procurou-se rastrear o impacto da patogênese orovalvar e das principais variáveis envolvidas no tratamento cirúrgico, capazes de alterar os resultados pós-operatórios da série de pacientes analisados. Os parâmetros intra-operatórios, como os tempos médios de circulação extracorpórea e de isquemia miocárdica revelaram valores muito próximos para os dois grupos confrontados, possivelmente, refletindo, de forma indireta, não haver maior complexidade na abordagem cirúrgica requerida por um ou outro tipo de lesão valvar. Da mesma forma, em relação às variáveis - óbito, implante de marcapasso, administração de antiarrítmico e/ou de anticoagulantes - discriminadas no pós-operatório imediato e tardio - não se verificaram diferenças significativas entre os pacientes com lesão valvar reumática ou degenerativa. As médias dos tempos de internação e de follow up, à semeIhança do que ocorreu com as variáveis intra-operatórias, também equipararam-se.

Observando-se a distribuição da série em estudo nas classes funcionais I, II, III e IV (critérios da NYHA), referente ao pós-operatório tardio, constata-se que pertencer ao grupo $R$ ou $D$ não afetou de forma significativa o resultado final do tratamento. 
Kalil R A K, Cunha B, Albrecht A S, Moreno P, Abrahão R, Prates PR, Sant'Anna J R M, Nesralla I A - Análise comparativa dos resultados da cirurgia do labirinto (Cox-maze) para fibrilação atrial crônica em pacientes com doença valvar mitral reumática ou degenerativa. Rev Bras Cir Cardiovasc 1999; 14 (3): 191-99.

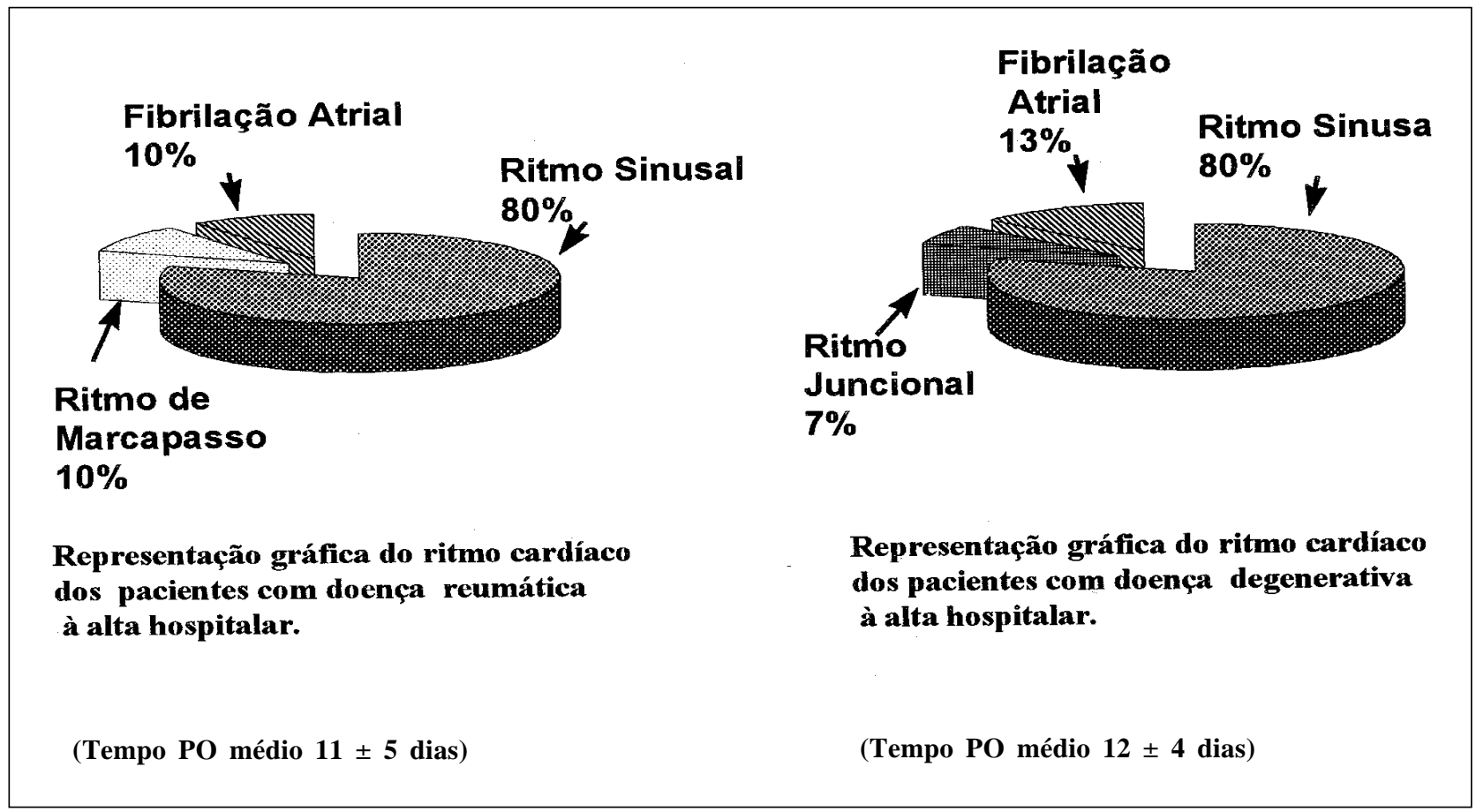

Fig. 1

Salienta-se o contraste entre a maior concentração de pacientes, tanto reumáticos como degenerativos, na classe III pré-operatória, e a maior parte desse contingente populacional incidindo na classe II após o tratamento cirúrgico.
$\mathrm{Na}$ análise ecocardiográfica realizada por ocasião da última avaliação, as médias de diâmetro de átrio esquerdo exibiram valores aproximados para as duas populações, sendo possível inferir que a degeneração provocada pela atividade inflamatória

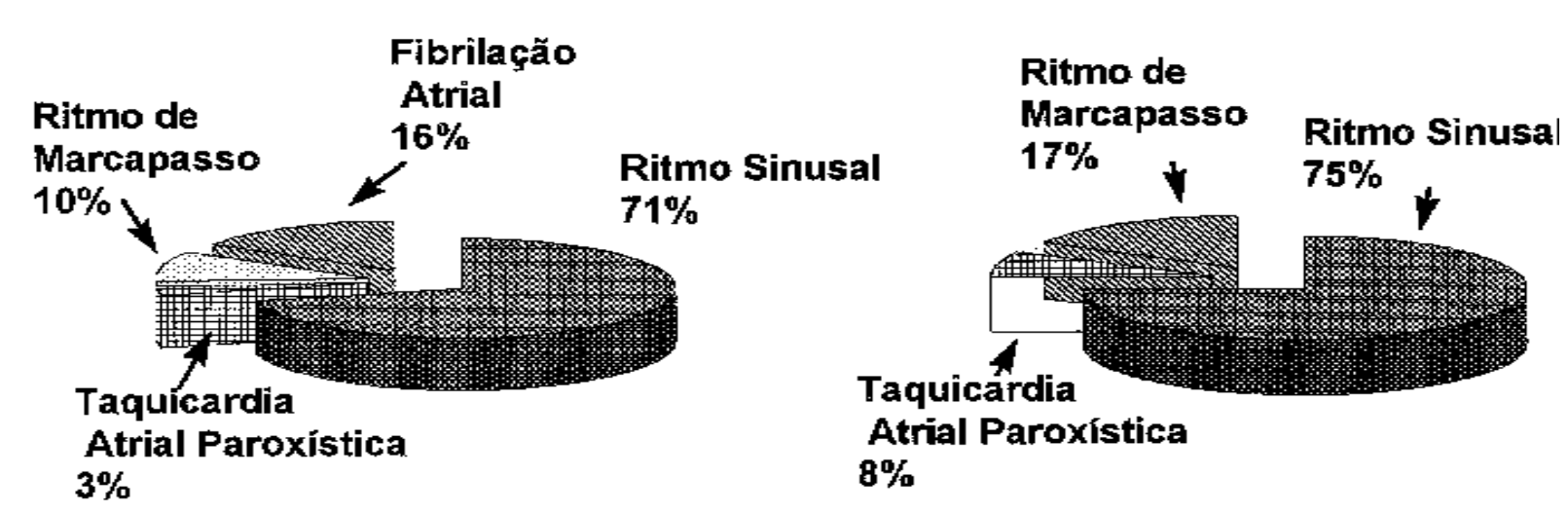

Representação gráfica do ritmo cardiaco dos pacientes com doença remuática na última avaliação pós-operatória.

Representação gráfica do ritmo ca rdía co dos pacientes com doença degenerativa na última avaliação pós-operatória.

(Tempo PO médio 17,4 \pm 14,1 meses)

(Tempo PO médio 18,5 \pm 12,1 meses) 
Kalil R A K, Cunha B, Albrecht A S, Moreno P, Abrahão R, Prates PR, Sant'Anna J R M, Nesralla I A - Análise comparativa dos resultados da cirurgia do labirinto (Cox-maze) para fibrilação atrial crônica em pacientes com doença valvar mitral reumática ou degenerativa. Rev Bras Cir Cardiovasc 1999; 14 (3): 191-99.

GRÁFICO 1

CLASSE FUNCIONAL PRÉ E PÓS-OPERATÓRIA (NYHA) NOS PACIENTES COM DOENÇA VALVAR REUMÁTICA E DEGENERATIVA

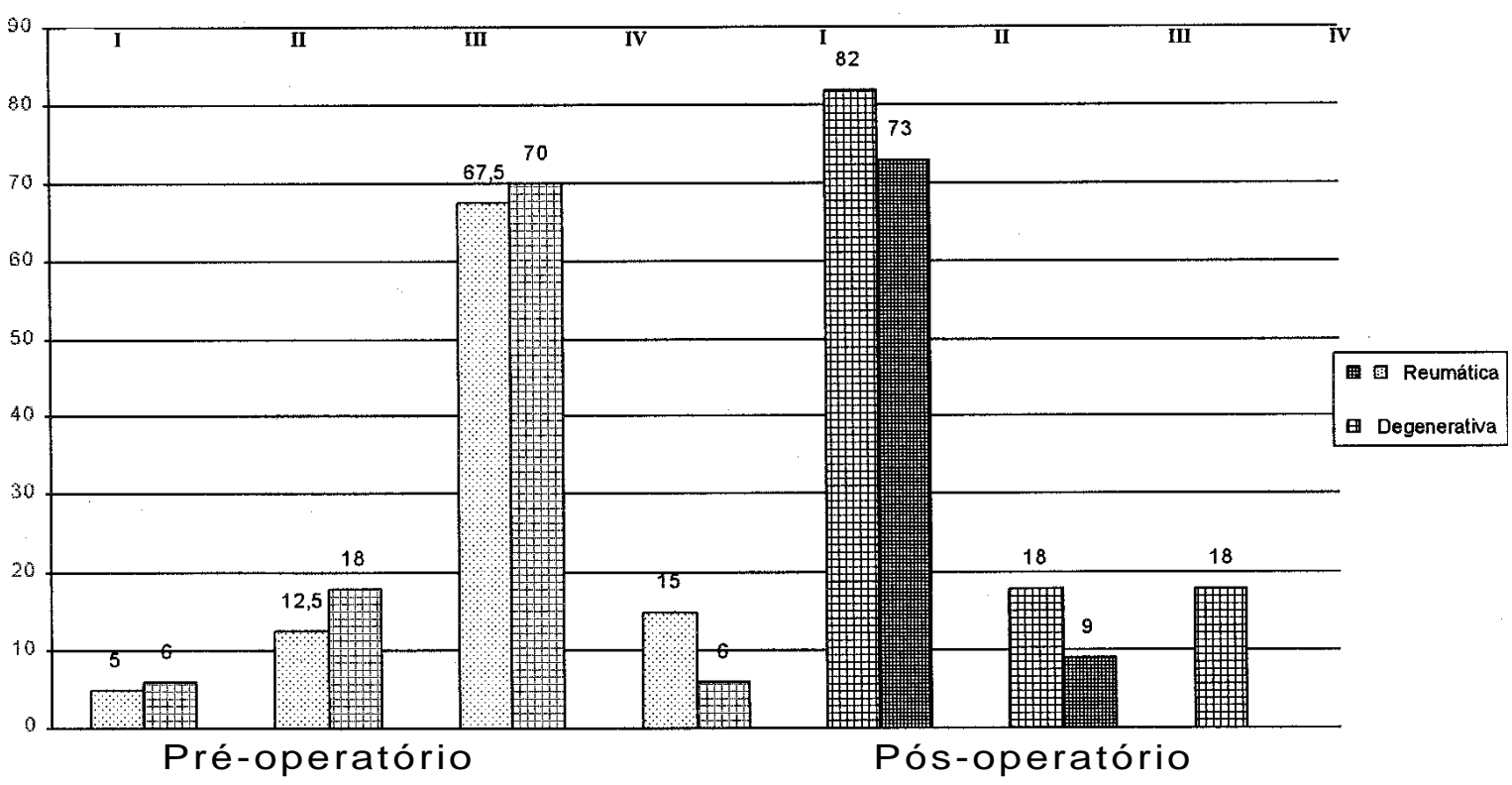

* Excluídos os óbitos hospitalares e o porcentual de pacientes

para o qual não foi possível obter informação sobre a classe funcional pós-operatória

reumática, necessariamente, não determina a ocorrência de cavidades atriais esquerdas maiores do que aquelas geradas pela doença valvar degenerativa - o que se pode observar no estudo de FUKADA et al. (7) onde comparação desse parâmetro entre uma população de pacientes reumáticos e não-reumáticos também não exibiu diferença estatística; embora, ao contrário do que foi evidenciado no presente trabalho, os valores médios do diâmetro da cavidade atrial dos pacientes reumáticos foi superior aos referentes ao outro Grupo. Globalmente, as dimensões médias de átrio esquerdo foram compatíveis com aquelas consideradas preditivas de sucesso $(10,12)$ na recuperação do ritmo atrial após o Procedimento do Labirinto na população estudada por KOBAYASHI et al. (12). As médias das frações de ejeção, também, foram semelhantes, situando-se dentro do intervalo previsto para os indivíduos adultos normais.

Os parâmetros ergométricos, para avaliação da resposta cronotrópica ao exercício, mensurados ao cabo de um período de follow up equivalente a 16,8 meses para os pacientes reumáticos e a 16,4 meses para o Grupo $D$, representaram as únicas variáveis para as quais foi possível encontrar diferenças estatisticamente significativas entre os pacientes comparados. Assim, a resposta cronotrópica (RC) ao exercício considerada intermediária (entre
$75 \%$ e $85 \%$ da freqüência cardíaca máxima calculada) foi o desempenho ergométrico prevalente no Grupo de pacientes com lesão degenerativa; ao passo que, a RC classificada como inadequada (menor do que $75 \%$ da freqüência cardíaca máxima) prevaleceu nos pacientes reumáticos. É interessante notar, entretanto, que o número de respostas cronotrópicas normais ou adequadas foi maior no Grupo reumático, quando este dado é comparado à soma das respostas intermediária e inadequada de ambos os Grupos. Deve-se ressaltar que como os períodos médios de pós-operatório à ergometria ultrapassaram 12 meses, consideramos que os pacientes analisados já haviam recuperado o tônus autonômico, claramente ausente nos primeiros meses de pós-operatório em função da denervação do SNA provocado pelas múltiplas incisões nos átrios (13). Dessa forma, consideramos não ter esse fator interagido com a competência cronotrópica dos pacientes.

FUKADA et al. (7), em recente publicação, ao estabelecer uma comparação entre o porcentual de pacientes em que foi possível o retorno a ritmo sinusal atrial após a Operação do Labirinto associada à correção valvar mitral e aqueles em que houve manutenção da fibrilação atrial, encontraram diferenças significativas em relação à fisiopatogênese da doen- 
Kalil R A K, Cunha B, Albrecht A S, Moreno P, Abrahão R, Prates PR, Sant'Anna J R M, Nesralla I A - Análise comparativa dos resultados da cirurgia do labirinto (Cox-maze) para fibrilação atrial crônica em pacientes com doença valvar mitral reumática ou degenerativa. Rev Bras Cir Cardiovasc 1999; 14 (3): 191-99.

ça cardíaca estrutural. Na série analisada, constituída por 22 pacientes com estenose mitral de etiologia reumática (Grupo I) e 7 com lesão valvar de origem não-reumática (Grupo II), o índice de recuperação do ritmo atrial alcançou 59\%, estando contida nesse porcentual a população de pacientes não-reumáticos acrescida de 10 pacientes do Grupo I. Os 12 pacientes para as quais não foi possível reversão a ritmo atrial apresentavam acometimento valvar de etiologia reumática.

Em relato anterior, KOSAKAI et al. (8) também creditaram à natureza predominantemente reumática das lesões valvares descritas em sua série de pacientes o menor porcentual de conversão a ritmo atrial detectado, embora essa associação tenha se mostrado negativa na análise comparativa dos resultados pós-operatórios.

KOBAYASHI et al. (12) investigando critérios de seleção que possam incrementar o índice de sucesso cirúrgico observaram que a incidência de doença valvar reumática foi significativamente mais alta no Grupo de pacientes que manifestou maior índice de insucesso em relação à recuperação do ritmo atrial/sinusal.

Em contrapartida, em trabalhos como os de KAMATA et al. (10) e KAWAGUCHI et al. (9) não foi possível atribuir à patogênese da lesão valvar nenhum impacto prognóstico significativo, devendo-se ainda salientar que no estudo de FUKADA et al. (7) anteriormente citado, algumas modificações na técnica cirúrgica foram apontadas como protagonistas nos resultados menos promissores alcançados pelos pacientes ${ }^{(14)}$.

Neste estudo, os porcentuais de retorno a ritmo sinusal foram bastante satisfatórios para os dois Grupos analisados à alta hospitalar e no momento da última avaliação pós-operatória; porém tanto em relação a esse como aos outros tipos de ritmo que compuseram o perfil eletrocardiográfico dos pacientes - ritmo de marcapasso, fibrilação atrial, ritmo juncional e taquicardia atrial paroxística - a etiologia reumática ou degenerativa não exerceu qualquer impacto, pelo menos de forma significativa, no ritmo cardíaco pós-operatório.

Um outro aspecto a ser comentado é a ocorrência de uma relativa manutenção do perfil eletrocardiográfico dos pacientes ao longo do período de follow-up, demonstrando a tendência, em qualquer uma das populações investigadas, à estabilidade.

\section{CONCLUSÃO}

A etiologia da lesão valvar - reumática ou degenerativa - não afeta a morbimortalidade imediata e tardia dos pacientes com valvopatia mitral submetidos à Cirurgia do Labirinto e à correção mitral simultaneamente. A recuperação do ritmo sinusal/atrial e a presença de arritmias no pós-operatório foi semelhante em ambos os Grupos. Apenas em relação à resposta cronotrópica ao exercício, verificou-se uma tendência, no Grupo degenerativo, a exibir valores menores. Portanto, a etiologia da lesão valvar não parece influir nos resultados da Cirurgia do Labirinto para correção da fibrilação atrial crônica.

RBCCV 44205-460

Kalil R A K, Cunha B, Albrecht A S, Moreno P, Abrahão R, Prates P R, Sant'Anna J R M, Nesralla I A Comparative results of maze procedure for chronic atrial fibrillation in rheumatic and degenerative mitral valve disease. Rev Bras Cir Cardiovasc 1999; 14 (3): 191-99.

ABSTRACT: The results of maze procedure in rheumatic mitral valve disease are subject to debate. This paper reports medium term results in rheumatic and degenerative mitral valve surgery associated with maze procedure. From 1994 to 1997, 57 patients were operated on. They were divided into two Groups; R (rheumatic) 40 patients and D (degenerative) 17. Group R included 8 (20\%) males and $32(80 \%)$ females. Group D: $8(47 \%)$ males and $9(53 \%)$ females (NS). Age in $R=47 \pm 11$ and $D 54 \pm 17$ years $(p<0.05)$. Left atrial size was 6.1 $\pm 1.1 \mathrm{~cm}$ in R and $5.9 \pm 1.2$ in D (NS). There were 3 hospital deaths, $1(2.5 \%)$ in R and $2(12 \%)$ in D. One D patient $(7 \%)$ died late. Pacemakers were implanted in $4(10 \%) R$ and $2(17 \%)$ in $D(N S)$. There were no significant differences regarding surgical duration of perfusion or myocardial ischemia, antiarrhythmic medications, immediate or late cardiac rythms and occurrence of arrythmias. Ergometric evaluation for measuring chronotropic response revealed a normal response in $6(25 \%) \mathrm{R}$ and $1(10 \%)$ in $\mathrm{D}$ at a mean of 16.6 months PO. In the lower response group, $3(12.5 \%) R$ and $6(60 \%) D$ had intermediate values $(p=0.009)$ and $15(62.5 \%)$ $R$ versus $3(30 \%)$ D had values below $75 \%$ of expected heart rate $(p=0.09)$. Rheumatic or degenerative ethiology of mitral valve disease does not influence the results of maze procedure in this patient population. Mean chronotropic response to exercise tends to be lower in degenerative patients.

DESCRIPTORS: Mitral valve, surgery. Atrial fibrillation, surgery. Arrhythmia, sinus, surgery. Rheumatic heart disease. 
Kalil R A K, Cunha B, Albrecht A S, Moreno P, Abrahão R, Prates PR, Sant'Anna J R M, Nesralla I A - Análise comparativa dos resultados da cirurgia do labirinto (Cox-maze) para fibrilação atrial crônica em pacientes com doença valvar mitral reumática ou degenerativa. Rev Bras Cir Cardiovasc 1999; 14 (3): 191-99.

\section{REFERÊNCIAS BIBLIOGRÁFICAS}

1. Cox J L, Schuessler R B, D’Agostino H J Jr et al. - The surgical treatment of atrial fibrillation. III: Development of a definitive surgical procedure. $J$ Thorac Cardiovasc Surg 1991; 101: 569-83.

2. Zipes D P - Specific arrhythmias: diagnosis and treatment. In: Braunwald E, ed. Heart disease: a textbook of cardiovascular medicine. Philadelphia: WB Saunders Company, 1992: 682.

3. Melo J Q, Neves J P, Abecasis L M, Adragão $P$, Ribeiras $R$, Seabra-Gomes R - Operative risks of the maze procedure associated with mitral valve surgery. Cardiovasc Surg 1997; 5: 112-6.

4. Cox J L, Boineau J P, Schuessler R B, Kater K M, Lappas D G - Five-year experience with the maze procedure for atrial fibrillation. Ann Thorac Surg 1993; 56: 814-24.

5. Chua Y L, Schaff H V, Orszulak T A, Morris J J - Outcome of mitral valve repair in patients with preoperative atrial fibrillation: should the maze procedure be combined with mitral valvuloplasty? J Thorac Cardiovasc Surg 1994; 107: 408-15.

6. Izumoto H, Kawazoe K, Kitahara H, Kamata J - Operative results after the Cox/maze procedure combined with a mitral valve operation. Ann Thorac Surg 1998; 66: 800-4.

7. Fukada J, Morishita K, Komatsu $\mathrm{K}$ et al. - Is atrial fibrillation resulting from rheumatic mitral valve disease a proper indication for the maze procedure? Ann Thorac Surg 1998; 65: 1566-70.
8. Kosakai $\mathrm{Y}$, Kawaguchi A T, Isobe F et al. - Cox maze procedure for chronic atrial fibrillation associated with mitral valve disease. J Thorac Cardiovasc Surg 1994; 108: 1049-55.

9. Kawaguchi A T, Kosakai $\mathrm{Y}$, Isobe $\mathrm{F}$ et al. - Factors affecting rhythm after the maze procedure for atrial fibrillation. Circulation 1996; 94(9Suppl): II 139-42.

10. Kamata J, Kawazoe K, Izumoto $\mathrm{H}$ et al. - Predictors of sinus rhythm restoration after Cox maze procedure concomitant with other cardiac operations. Ann Thorac Surg 1997; 64: 394-8.

11. Cox J L, Jaquiss R D, Schuessler R B, Boineau J P Modification of the maze procedure for atrial flutter and atrial fibrillation. II: Surgical technique of the maze III procedure. J Thorac Cardiovasc Surg 1995; 110: 485-95.

12. Kobayashi J, Kosakai Y, Nakano K, Sasako Y, Eishi K, Yamamoto $F$ - Improved sucess rate of the maze procedure in mitral valve disease by new criteria for patients' selection. Eur J Cardiothorac Surg 1998; 13: 247-52.

13. Vogt P R, La Rocca H P, Candinas R et al. - Temporary loss of cardiac autonomic innervation after the maze procedure. Eur J Cardiothorac Surg 1997; 12: 75-81.

14. Cox J L - Invited Commentary. In: Fukada J, Morishita $\mathrm{K}$, Komatsu $\mathrm{K}$ et al. - Is atrial fibrillation resulting from rheumatic mitral valve disease a proper indication for the maze procedure? Ann Thorac Surg 1998; 65: 1566-70. 